\title{
Chiral effective field theory for nuclear matter including long- and short-range multi-nucleon interactions
}

\author{
A. Lacour ${ }^{1, a}$, J. A. Oller² , and U.-G. Meißner ${ }^{1,3}$ \\ 1 Helmholtz-Institut für Strahlen- und Kernphysik (Theorie) and Bethe Center for Theoretical Physics, \\ Universität Bonn, D-53115 Bonn, Germany \\ 2 Departamento de Física, Universidad de Murcia, E-30071 Murcia, Spain \\ 3 Institut für Kernphysik, Institute for Advanced Simulation and Jülich Center for Hadron Physics, \\ Forschungszentrum Jülich, D-52425 Jülich, Germany
}

\begin{abstract}
We review on a novel chiral power counting scheme for in-medium chiral perturbation theory with nucleons and pions as degrees of freedom. It allows for a systematic expansion taking into account local as well as pion-mediated inter-nucleon interactions. Based on this power counting, one can identify classes of nonperturbative diagrams that require a resummation. As a method for performing those resummations we review on the techniques of Unitary Chiral Pertubation Theory for nucleon-nucleon interactions. We then apply both power counting and non-perturbative methods to the example of calculating the pion self-energy in asymmetric nuclear matter up-to-and-including next-to-leading order. It is shown that the leading corrections involving in-medium nucleon-nucleon interactions cancel between each other at given chiral orders.
\end{abstract}

\section{Introduction}

An interesting achievement in nuclear physics would be the calculation of atomic nuclei and nuclear matter properties from microscopic inter-nucleon forces in a systematic and controlled way. This is a non-perturbative problem involving the strong interactions. In the last decades, Effective Field Theory (EFT) has proven to be an indispensable tool to accomplish such an ambitious goal. In this work we employ Chiral Perturbation Theory (CHPT) to nuclear systems [3-5], with nucleons and pions as the pertinent degrees of freedom. For the lightest nuclear systems with two, three and four nucleons, it has been successfully applied [6]. For heavier nuclei one common procedure is to employ the chiral nucleon-nucleon (NN) potential derived in CHPT combined with standard many-body methods, sometimes supplied with renormalization group techniques [7]. In ref.[1] we have recently derived a chiral power counting in nuclear matter that takes into account local multi-nucleon interactions simultaneously to the pionnucleon interactions. Many present applications of CHPT to nuclei and nuclear matter only consider meson-baryon chiral Lagrangians (see e.g. [6] for a summary), without constraints from free NN scattering. In ref.[2] the techniques of Unitary Chiral Perturbation Theory (UCHPT) $[8,9]$ have been applied to perform the resummation of the non-pertubative nature of the $\mathrm{NN}$-interaction to vacuum phase shifts as well as to in-medium $\mathrm{NN}$-scattering up to next-to-leading order (NLO). Our novel power counting

\footnotetext{
a e-mail: lacour@hiskp.uni-bonn.de
}

is applied in refs.[1,2] to the problem of calculating the pion self-energy in asymmetric nuclear matter at NLO. ${ }^{1}$

\section{Chiral Power Counting}

Ref.[18] considers the ground state of nuclear matter which, under the action of any time dependent operator at asymptotic times, behaves as Fermi seas of nucleons. The Fermi seas are filled states up to a limiting Fermi momentum $\xi_{i_{3}}=\left(3 \pi^{2} \rho_{i_{3}}\right)^{1 / 3}$, with $\rho_{i_{3}}$ the density of the corresponding Fermi sea. Ref.[18] also establishes the concept of an "in-medium generalized vertex" (IGV). Such type of vertices result because one can connect several bilinear vacuum vertices through the exchange of baryon propagators with the flow through the loop of one unit of baryon number, contributed by the nucleon Fermi seas. At least one is needed, otherwise we would have a vacuum closed nucleon loop that in a low energy effective field theory is buried in the higher order counterterms. It was also stressed in ref.[12] that within a nuclear environment a nucleon propagator could have a "standard" or "non-standard" chiral counting. To see this, note that a soft momentum $Q \sim p$, related to pions or external sources can be associated to any of the vertices. Denoting by $k$ the on-shell four-momenta associated with one Fermi sea insertion in the IGV, the

1 This problem is tightly connected with that of pionic atoms $[10,11]$ due to the relation between the pion self-energy and the pion-nucleus optical potential. For recent calculations see [1217]. 
four-momentum running through the $j^{\text {th }}$ nucleon propagator can be written as $p_{j}=k+Q_{j}$. If $Q_{j}^{0}=O\left(m_{\pi}\right)=O(p)$, one has the standard counting so that the baryon propagator scales as $O\left(p^{-1}\right)$. However, if $Q_{j}^{0}$ is of the order of the kinetic nucleon energy in the nuclear medium then the nucleon propagator should be counted as $O\left(p^{-2}\right)$. This is referred to as the "non-standard" case in ref.[12]. In order to treat chiral Lagrangians with an arbitrary number of baryon fields (bilinear, quartic, etc) ref.[1] considered firstly bilinear vertices like in refs.[12,18], but now the additional exchanges of heavy meson fields of any type are allowed. They should be considered merely as auxiliary fields that allow one to find a tractable representation of the multi-nucleon interactions that result when the masses of the heavy mesons tend to infinity. Consequently a heavy meson propagator is counted as $O\left(p^{0}\right)$ due to their large masses. On the other hand, ref.[1] takes the non-standard counting case from the start and any nucleon propagator is considered as $O\left(p^{-2}\right)$. In this way no diagram, whose chiral order is actually lower than expected if the nucleon propagators were counted assuming the standard rules, is lost. In the following $m_{\pi} \sim k_{i_{3}} \sim O(p)$ are taken of the same chiral order, and are considered much smaller than a hadronic scale $\Lambda_{\chi}$ of several hundred $\mathrm{MeV}$ that results by integrating out all other particle types, including nucleons with larger three-momentum, heavy mesons and nucleon isobars [5]. The final formula obtained in ref.[1] for the chiral order $p^{v}$ of a given diagram is

$$
\begin{aligned}
v & =4-E+\sum_{i=1}^{V_{\pi}}\left(n_{i}+\ell_{i}-4\right)+\sum_{i=1}^{V}\left(d_{i}+w_{i}-1\right)+\sum_{i=1}^{m}\left(v_{i}-1\right)+\sum_{i=1}^{V_{\rho}} v_{i} \\
& =4-E+\sum_{i=1}^{V_{\pi}}\left(n_{i}+\ell_{i}-4\right)+\sum_{i=1}^{V}\left(d_{i}+v_{i}+w_{i}-2\right)+V_{\rho} .
\end{aligned}
$$

where $E$ is the number of external pion lines, $n_{i}$ is the number of pion lines attached to a vertex without baryons, $\ell_{i}$ is the chiral order of the latter with $V_{\pi}$ its total number. In addition, $d_{i}$ is the chiral order of the $i^{\text {th }}$ vertex bilinear in the baryonic fields, $v_{i}$ is the number of mesonic lines attached to it, $w_{i}$ that of only the heavy lines, $V$ is the total number of bilinear vertices, $V_{\rho}$ is the number of IGVs and $m$ is the total number of baryon propagators minus $V_{\rho}$, so $V=V_{\rho}+m$. It is important to stress that $v$ given in eq.(2.1) is bounded from below [1]. Because of the last term in eq.(2.1) adding a new IGV to a connected diagram increases the counting at least by one unit because $v_{i} \geq 1$. The number $v$ given in eq.(2.1) represents a lower bound for the actual chiral power $\mu$ of a diagram, so that $\mu \geq v$. The real chiral order of a diagram might be different from $v$ because the nucleon propagators are counted always as $O\left(p^{-2}\right)$ in eq.(2.1), while for some diagrams there could be propagators that follow the standard counting. Eq.(2.1) implies the following conditions for augmenting the number of lines in a diagram without increasing the chiral power by adding i) pionic lines attached to mesonic vertices, $\ell_{i}=n_{i}=2$, ii) pionic lines attached to meson-baryon vertices, $d_{i}=v_{i}=1$ and iii) heavy mesonic lines attached to bilinear vertices, $d_{i}=0, w_{i}=1$.

\section{Non-pertubative methods}

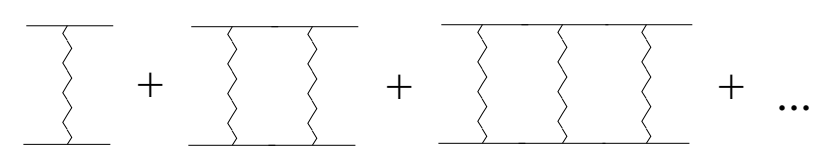

Fig. 1. Resummation of the two-nucleon reducible diagrams. This is referred in the text also as a resummation of the right-hand or unitarity cut. A wiggly line represents the interaction kernel $N_{J I}(\ell, \bar{\ell}, S)$.

Refs. $[4,5]$ argued that due to the large nucleon mass the two-nucleon reducible diagrams should be resummed, as it is schematically depicted in fig.1. The wiggly line is understood as a local $\mathrm{NN}$ interaction plus the exchange of a pion (fig. 2 left-hand side). Both are of order $O\left(p^{0}\right)$, as can be deduced from the Langrians in subsec.3.2. Accourding to ref.[1], the nucleon propagators in the diagrams of fig. 1 follow the non-standard counting and each of them is $O\left(p^{-2}\right)$. The two-nucleon propagators altogether are $O\left(p^{-4}\right)$, multiplied by the $O\left(p^{4}\right)$ contribution from the measure of the loop integral and the $O\left(p^{0}\right)$ wiggly lines produces an $O\left(p^{0}\right)$ contribution. This does not increase the chiral order and the series of diagrams in fig. 1 must be resummed.

\subsection{Unitary CHPT}

We follow the techniques of UCHPT $[2,8,9]$ that performs the resummation partial wave by partial wave. ${ }^{2}$ The master equation for UCHPT is

$$
T_{J I}(\ell, \bar{\ell}, S)=\left[I+N_{J I}(\ell, \bar{\ell}, S) \cdot g\right]^{-1} \cdot N_{J I}(\ell, \bar{\ell}, S) .
$$

It results by performing a once subtracted dispersion relation of the inverse of a partial wave amplitude. $T_{J I}$ is the scattering amplitude and $N_{J I}$ is the interaction kernel with quantum numbers total isospin $I$, total spin $S$, out- and ingoing orbital angular momenta $\ell, \bar{\ell}$ and total angular momentum $J . g$ is called the unitarity loop that corresponds to the divergent integral [2]

$$
g(A)=-m \int \frac{d^{3} k}{(2 \pi)^{3}} \frac{1}{k^{2}-A-i \epsilon} .
$$

In this way the effects of the nucleon mass associated with the two-nucleon reducible diagrams are taken into account. On the contrary, in a plain chiral calculation of a NN partial wave these effects are not resummed. But we can match the latter with the expansion of eq.(3.1) up to the same number of two-nucleon reducible loops [2].

$$
T_{J I}(\ell, \bar{\ell}, S)=N_{J I}(\ell, \bar{\ell}, S)-N_{J I}(\ell, \bar{\ell}, S) \cdot g \cdot N_{J I}(\ell, \bar{\ell}, S)+\ldots
$$

2 This is different from solving a Lippmann-Schwinger equation, where the full scattering amplitude is calculated and the partial waves projection is performed afterwards. 


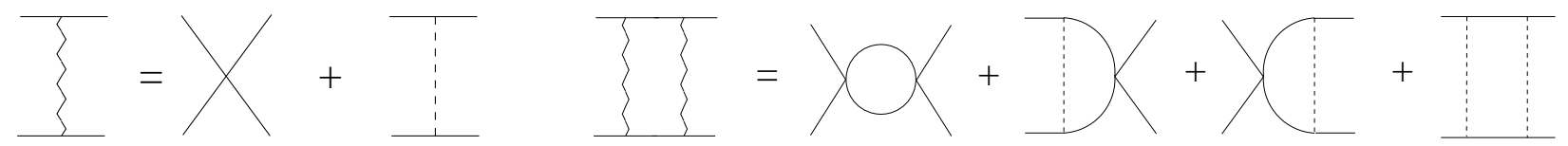

Fig. 2. The exchange of a wiggly line between two nucleons indicates the sum of the local and the one-pion exchange. A one-wiggle exchange corresponds to the leading order (LO) interaction kernel $N_{J I}^{(0)}$, whereas the two-wiggle exchange box diagram corresponds to $L_{J I}^{(1)}$.

Notice that in this expansion each factor of the kernel $N_{J I}$ multiplies the loop function $g$ with its value on-shell. In addition one has the chiral expansion of the interaction kernel

$$
N_{J I}=\sum_{m=0}^{\infty} N_{J I}^{(m)}=N_{J I}^{(0)}+N_{J I}^{(1)}+O\left(p^{2}\right),
$$

where the chiral order is indicated by the superscript. In order to extract $N_{J I}^{(n)}$, one has to match the $O\left(p^{n}\right)$ chiral calculation of a NN partial wave comprising at most $n$ twonucleon reducible loops with eq.(3.3) [2]

$$
N_{J I}^{(0)}+N_{J I}^{(1)}-N_{J I}^{(0)} \cdot g \cdot N_{J I}^{(0)}+O\left(p^{2}\right)=N_{J I}^{(0)}+L_{J I}^{(1)}+O\left(p^{2}\right) .
$$

On the right hand side of eq.(3.5) the chiral calculation is given, which is diagramatically shown in fig.2: $N_{J I}^{(0)}$ (left diagram) is the $O\left(p^{0}\right)$ CHPT calculation, without any twonucleon reducible loop and $L_{J I}^{(1)}$ (right diagram) is the $O\left(p^{1}\right)$ contribution with one two-nucleon reducible loop, respectively for a given partial. Here each two-nucleon reducible loop calculated in CHPT ist counted as $O(p)$ as suggested by eq.(3.2). Thus the $O(p)$ contribution to the interaction kernel reads [2]

$$
N_{J I}^{(1)}=L_{J I}^{(1)}+N_{J I}^{(0)} \cdot g \cdot N_{J I}^{(0)},
$$

which corresponds to the difference between a full calculation of a two-nucleon reducible loop and the result obtained by factorizing the vertices on-shell. It is consistent with the counting as the large nucleon mass factor drops in the difference. This fact is incorporated in the interaction kernel $N_{J I}$, which is then improved order by order.

\subsection{Fixing vacuum properties}

For the following evaluations we employ the $O(p)$ and $O\left(p^{2}\right)$ Heavy Baryon CHPT (HBCHPT) Lagrangians [19]

$$
\begin{aligned}
\mathcal{L}_{\pi N}^{(1)} & =\bar{N}\left(i D_{0}-\frac{g_{A}}{2} \sigma \cdot \mathbf{u}\right) N \\
\mathcal{L}_{\pi N}^{(2)} & =\bar{N}\left(\frac{1}{2 M} \mathbf{D} \cdot \mathbf{D}+i \frac{g_{A}}{4 M}\left\{\sigma \cdot \mathbf{D}, u_{0}\right\}+2 c_{1} m_{\pi}^{2}\left(U+U^{\dagger}\right)\right. \\
& \left.+\left(c_{2}-\frac{g_{A}^{2}}{8 M}\right) u_{0}^{2}+c_{3} u_{\mu} u^{\mu}\right) N+\ldots
\end{aligned}
$$

$N$ is the two component field of the nucleons, $g_{A}$ the axial pion-nucleon coupling and $D_{\mu}=\partial_{\mu}+\Gamma_{\mu}$ the covariant chiral derivative, with $\Gamma_{\mu}=\left[u^{\dagger}, \partial_{\mu} u\right]$. The pion fields $\pi(x)$ enter in the matrix $u=\exp (i \tau \cdot \pi / 2 f)$, in terms of which $u_{\mu}=i\left\{u^{\dagger}, \partial_{\mu} u\right\}$ and $U=u^{2}$, with $f=92.4 \mathrm{MeV}$ the pion decay constant in the $\operatorname{SU}(2)$ chiral limit. The $c_{i}$ are chiral low-energy constants whose values are fitted from phenomenology [19]. Accourding to $[4,5]$ the lowest order amplitudes for the two-nucleon irreducible diagrams, are given by the quartic nucleon Lagrangian without quark masses or derivatives

$$
\mathcal{L}_{N N}^{(0)}=-\frac{1}{2} C_{S}(\bar{N} N)(\bar{N} N)-\frac{1}{2} C_{T}(\bar{N} \sigma N)(\bar{N} \sigma N)
$$

and the one-pion exchange with the lowest order pion-nucleon coupling. The scattering amplitudes that follow these Lagrangian terms can be found in ref.[2].

Calculating the integral of the unitarity loop eq.(3.2) explicitly, we employ a three-momentum cut-off $\Lambda$ [2]

$$
\begin{aligned}
g(A) & =-\frac{m}{2 \pi^{2}} \int_{0}^{\Lambda} d k-\frac{m A}{4 \pi^{2}} \int_{-\infty}^{\infty} \frac{d k}{k^{2}-A-i \epsilon} \\
& =-\frac{m \Lambda}{2 \pi^{2}}-\frac{i m \sqrt{A}}{4 \pi} .
\end{aligned}
$$

We now concentrate on fixing the constants $C_{S}$ and $C_{T}$ at leading order. For that we consider the S-wave NN scattering lengths $a_{t}$ and $a_{s}$ for triplet and singlet channels, respectively, at threshold. At threshold the one-pion exchanges vanishes, which also implies that the triplet $S$ partial wave is elastic, without mixing with the ${ }^{3} D_{1}$ partial wave. Using eq.(3.1) yields [2]

$$
\begin{aligned}
& C_{S}=\frac{\pi}{m} \frac{3 / a_{s}+1 / a_{t}-8 \Lambda / \pi}{\left(1 / a_{s}-2 \Lambda / \pi\right)\left(1 / a_{t}-2 \Lambda / \pi\right)}, \\
& C_{T}=\frac{\pi}{m} \frac{1 / a_{s}-1 / a_{t}}{\left(1 / a_{s}-2 \Lambda / \pi\right)\left(1 / a_{t}-2 \Lambda / \pi\right)} .
\end{aligned}
$$

One of the characteristics of NN scattering are the large absolute values of the $\mathrm{S}$-wave scattering lengths $a_{s}=$ $(-23.758 \pm 0.04) \mathrm{fm}$ and $a_{t}=(5.424 \pm 0.004) \mathrm{fm}$. For typical values $\Lambda \gg\left|1 / a_{s}\right|$, eq. (3.10) gives $\left|C_{S}\right| \simeq 2 \pi^{2} / m \Lambda \gg$ $\left|C_{T}\right|=O\left(\pi^{3} / a_{t} / m \Lambda^{2}\right)$. In this way, the low-energy constants $C_{S}$ and $C_{T}$ do not diverge for $a_{s}, a_{t} \rightarrow \infty$ because of the introduction of the new scale $\Lambda$ and they have a natural size. For a detailed discussion see ref.[2]. One has to stress that only local terms and one-pion exchange contributions enter in the calculation of $N_{J I}^{(0)}(\ell, \bar{\ell}, S)$. This is certainly too simplistic in order to properly describe the NN interactions as a function of energy up to pion production threshold. 


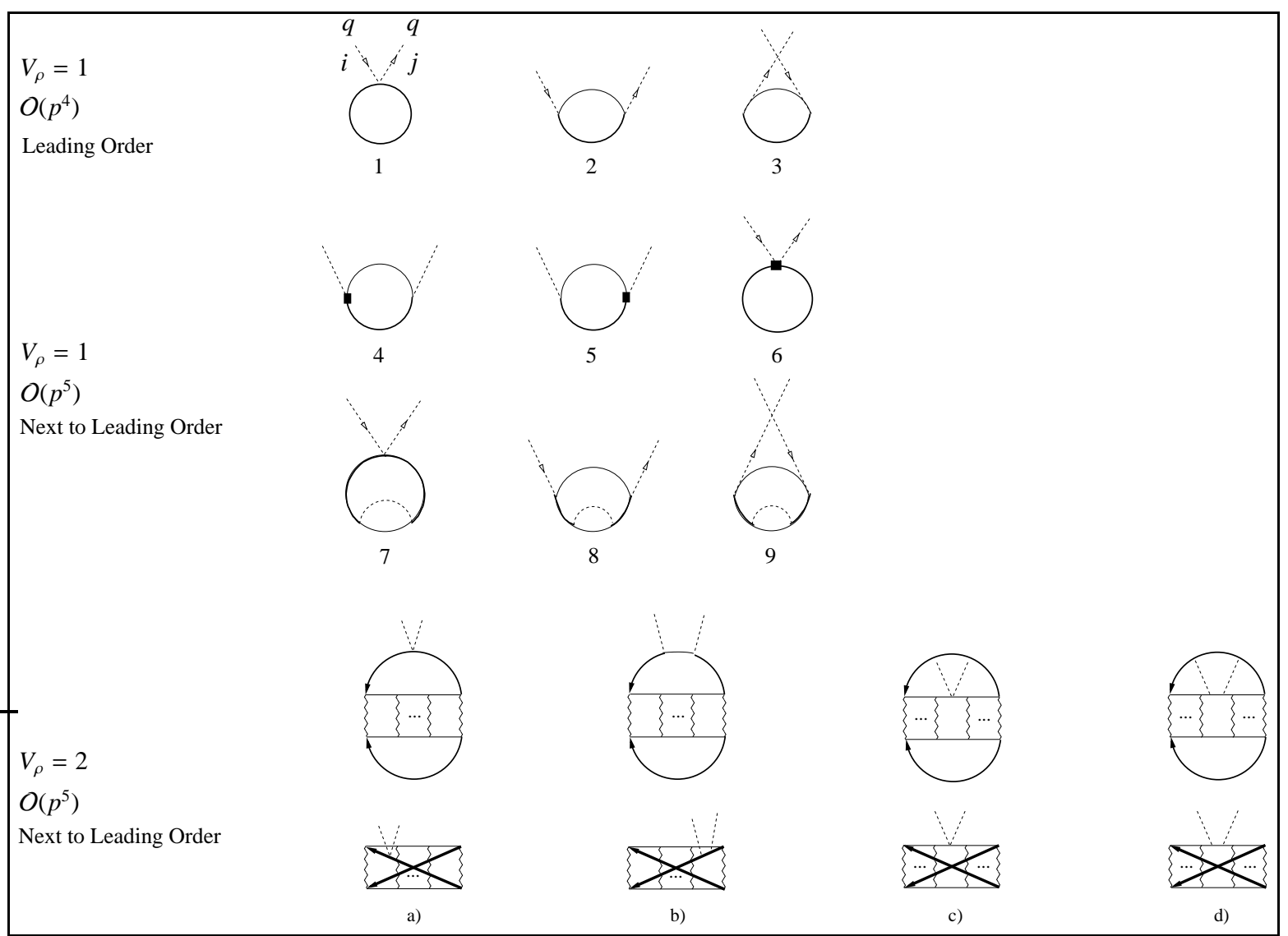

Fig. 3. Contributions to the in-medium pion self-energy up to NLO or $O\left(p^{5}\right)$. The pions are indicated by the dashed lines and the squares correspond to NLO pion-nucleon vertices. A wiggly line is the NN interaction kernel, that is iterated as indicated by the ellipsis.

\section{Pion-nucleon contributions to the pion self-energy}

Here, we apply the chiral counting given in eq.(2.1) to calculate the pion self-energy in the nuclear medium up to NLO or $O\left(p^{5}\right)$, with the different contributions shown in fig. 3. The nucleon propagator contains a particle part propagating above the Fermi surface and a hole part propagating below [20]. Using Cauchy's theorem this can be rewritten as a free-space and an in-medium part,

$$
\begin{aligned}
G_{0}(\mathbf{k})_{i_{3}} & =\frac{\theta\left(|\mathbf{k}|-\xi_{i_{3}}\right)}{k^{0}-\omega_{\mathbf{k}}+i \epsilon}+\frac{\theta\left(\xi_{i_{3}}-|\mathbf{k}|\right)}{k^{0}-\omega_{\mathbf{k}}-i \epsilon} \\
& =\frac{1}{k^{0}-\omega_{\mathbf{k}}+i \epsilon}+2 \pi i \delta\left(k^{0}-\omega_{\mathbf{k}}\right) \theta\left(\xi_{i_{3}}-|\mathbf{k}|\right)
\end{aligned}
$$

In this equation the subscript $i_{3}$ refers to the third component of isospin of the nucleon, which can be identified as $i_{3}=p$ for the proton and $i_{3}=n$ for the neutron. Our convention for the pion self-energy $\Sigma$, is such that the dressed pion propagators reads $i \Delta_{\pi}(q)=i /\left(q^{2}-m_{\pi}^{2}+\Sigma\right)$. The leading contribution to the pion self-energy corresponds to the diagrams $1\left(\Sigma_{1}\right)$ and $2+3\left(\Sigma_{2}\right)$, with [1]

$$
\begin{aligned}
& \Sigma_{1}=\frac{-i q^{0}}{2 f^{2}} \varepsilon_{i j 3}\left(\rho_{p}-\rho_{n}\right), \\
& \Sigma_{2}=\frac{i g_{A}^{2} \mathbf{q}^{2}}{2 f^{2} q^{0}} \varepsilon_{i j 3}\left(\rho_{p}-\rho_{n}\right)-\frac{g_{A}^{2}}{4 f^{2}} \frac{\left(\mathbf{q}^{2}\right)^{2}}{m q_{0}^{2}} \delta_{i j}\left(\rho_{p}+\rho_{n}\right),
\end{aligned}
$$

where the proton(neutron) density is given by $\rho_{p(n)}=$ $\xi_{p(n)}^{3} / 3 \pi^{2}$. Now, we move to the NLO contributions. The sum of the diagrams 4 and 5 gives the result [1]

$$
\Sigma_{3}=\frac{g_{A}^{2} \mathbf{q}^{2}}{2 m f^{2}}\left(\rho_{p}+\rho_{n}\right) \delta_{i j} .
$$

The diagram 6 of fig. 3 is given by [1]

$\Sigma_{4}=\frac{-2 \delta_{i j}}{f^{2}}\left(2 c_{1} m_{\pi}^{2}-q_{0}^{2}\left(c_{2}+c_{3}-\frac{g_{A}^{2}}{8 m}\right)+c_{3} \mathbf{q}^{2}\right)\left(\rho_{p}+\rho_{n}\right)$.

Next, let us consider the contributions to the pion selfenergy due to the nucleon self-energy from a one-pion loop as depicted in the diagrams 7-9 of fig.3. The one-pion loop nucleon self-energy can be written as,

$$
\Sigma^{\pi}=\frac{1+\tau_{3}}{2} \Sigma_{p}^{\pi}+\frac{1-\tau_{3}}{2} \Sigma_{n}^{\pi},
$$


with $\Sigma_{p}^{\pi}$ and $\Sigma_{n}^{\pi}$ the proton and nucleon self-energies due to the in-medium pion-nucleon loop. The contributions from the diagrams $7\left(\Sigma_{5}\right)$ and $8+9\left(\Sigma_{6}\right)$ are [1]

$$
\begin{aligned}
\Sigma_{5} & =\frac{q^{0}}{f^{2}} \varepsilon_{i j 3} \int \frac{d^{4} k}{(2 \pi)^{4}}\left(G_{0}(k)_{p} \frac{\partial \Sigma_{p}^{\pi}}{\partial k^{0}}-G_{0}(k)_{n} \frac{\partial \Sigma_{n}^{\pi}}{\partial k^{0}}\right) e^{i k^{0} \eta} \\
\Sigma_{6} & =\frac{-i g_{A}^{2}}{f^{2}} \frac{\mathbf{q}^{2}}{q^{0}} \varepsilon_{i j 3} \int \frac{d^{4} k}{(2 \pi)^{4}}\left(G_{0}(k)_{p} \frac{\partial \Sigma_{p}^{\pi}}{\partial k^{0}}-G_{0}(k)_{n} \frac{\partial \Sigma_{n}^{\pi}}{\partial k^{0}}\right) e^{i k^{0} \eta} \\
& +\frac{i g_{A}^{2}}{f^{2}} \frac{\mathbf{q}^{2}}{q_{0}^{2}} \delta_{i j} \int \frac{d^{4} k}{(2 \pi)^{4}}\left(G_{0}(k)_{p} \Sigma_{p}^{\pi}+G_{0}(k)_{n} \Sigma_{n}^{\pi}\right) e^{i k^{0} \eta}
\end{aligned}
$$

where $e^{i k^{0} \eta}$ with $\eta \rightarrow 0^{+}$is the convergence factor associated with any closed loop made up by a single nucleon line [20]. The last term in $\Sigma_{6}$ is a recoil correction of one order higher and we neglect it. The free pion-loop nucleon self-energy is calculated in HBCHPT, employing regularization in the $\overline{M S}-1$ scheme. Its derivative is $O\left(p^{2}\right)$ [1] so that when inserted in $\Sigma_{5}$ and $\Sigma_{6}$ it gives rise to an $O\left(p^{6}\right)$ contribution that we neglect in the present work. As shown in ref.[1] the in-medium contribution to the pion-loop nucleon self-energy is even further suppressed, being a contribution of $O\left(p^{7}\right)$ to the pion self-energy. As a result, $\Sigma_{5}$ and $\Sigma_{6}$ are at least $O\left(p^{6}\right)$.

\section{In-medium nucleon-nucleon scattering contributions}

We now consider those NLO contributions to the pion selfenergy in the nuclear medium that involve the $\mathrm{NN}$ interactions. They are depicted in the diagrams of the last two rows of fig.3, where the ellipsis indicate the iteration of the two-nucleon reducible loops. For the diagrams b) and d) of fig. 3 the pion lines can leave or enter the diagrams. Since $V_{\rho}=2$ in these contributions one needs only the NN scattering amplitude at $O\left(p^{0}\right)$ to match with our required precision at NLO. This amplitude is obtained by iterating an infinite ladder of two nucleon reducible loops, with full in-medium nucleon propagators. The tree level amplitudes obtained from the $O\left(p^{0}\right)$ Lagrangian with four nucleons [5] and from the one-pion exchange with the lowest order pion-nucleon coupling. The sum of both is represented diagrammatically in fig. 3 by the exchange of a wiggly line. This procedure would correspond in vacuum to the leading NN scattering amplitude according to refs. $[4,5]$.

\subsection{Cancelation of the leading nucleon-nucleon contributions [1]}

The diagrams a) and c) of fig. 3 involve the Weinberg-Tomozawa (WT) vertex while b) and d) contain the pole terms of pion-nucleon scattering. It is remarkable that these NLO contributions cancel pairwise. At leading order in the chiral counting the pole term has the same structure as the WT
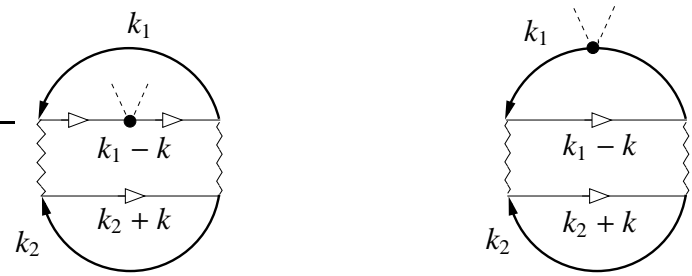

Fig. 4. Contribution to the pion self-energy with one two-nucleon reducible loop. The pion scatters inside/outside the loop.

term, with the summed vertex given by [1]

$$
-i \varepsilon_{i j k} \tau^{k} \frac{q^{0}}{2 f^{2}}\left(1-g_{A}^{2} \frac{\mathbf{q}^{2}}{q_{0}^{2}}\right)
$$

which is an isovector contribution. Note that the pole term also produces an isoscalar $1 / m$ recoil correction, which is one order higher. Let us denote by $\Sigma_{p(n)}^{N N}$ the proton (neutron) self-energy in the nuclear medium due to the $\mathrm{NN}$ interactions [1],

$$
\Sigma_{\alpha_{1}}^{N N}=-i \sum_{\alpha_{2}, \sigma_{2}} \int \frac{d^{4} k_{2}}{(2 \pi)^{4}} e^{i k_{2}^{0} \eta} G_{0}\left(k_{2}\right)_{\alpha_{2}} T_{\alpha_{1} \alpha_{2}}^{\sigma_{1} \sigma_{2}}\left(k_{1}, k_{2}\right),
$$

where we introduced the abbreviation

$$
T_{\alpha_{1} \alpha_{2}}^{\sigma_{1} \sigma_{2}}\left(k_{1}, k_{2}\right)=T_{N N}\left(k_{1} \sigma_{1} \alpha_{1} ; k_{2} \sigma_{2} \alpha_{2} \mid k_{1} \sigma_{1} \alpha_{1} ; k_{2} \sigma_{2} \alpha_{2}\right)
$$

for particle states with momentum $k_{i}$, spin $\sigma_{i}$ and isospin $\alpha_{i}$. The sum of diagram a) and the isovector part of b) of fig. 3 can then be written as [1]

$$
\begin{aligned}
\Sigma_{a}+\Sigma_{b}^{i v} & =-i \varepsilon_{i j 3} \frac{q^{0}}{2 f^{2}}\left(1-g_{A}^{2} \frac{\mathbf{q}^{2}}{q_{0}^{2}}\right) \sum_{\sigma_{1}, \sigma_{2}} \int \frac{d^{4} k_{1}}{(2 \pi)^{4}} \frac{d^{4} k_{2}}{(2 \pi)^{4}} \\
& \times e^{i k_{1}^{0} \eta} e^{i k_{2}^{0} \eta}\left[G_{0}\left(k_{1}\right)_{p} G_{0}\left(k_{2}\right)_{p} \frac{\partial}{\partial k_{1}^{0}} T_{p p}^{\sigma_{1} \sigma_{2}}\left(k_{1}, k_{2}\right)\right. \\
& \left.-G_{0}\left(k_{1}\right)_{n} G_{0}\left(k_{2}\right)_{n} \frac{\partial}{\partial k_{1}^{0}} T_{n n}^{\sigma_{1} \sigma_{2}}\left(k_{1}, k_{2}\right)\right]
\end{aligned}
$$

The isoscalar contribution of diagram b) reads

$$
\begin{aligned}
\sum_{b}^{i s} & =-i \delta_{i j} \frac{g_{A}^{2} \mathbf{q}^{2}}{2 f^{2} q_{0}^{2}} \sum_{\sigma_{1}, \sigma_{2}} \int \frac{d^{4} k_{1}}{(2 \pi)^{4}} \frac{d^{4} k_{2}}{(2 \pi)^{4}} e^{i k_{1}^{0} \eta} e^{i k_{2}^{0} \eta} \\
& \times\left[G_{0}\left(k_{1}\right)_{p} G_{0}\left(k_{2}\right)_{p} T_{p p}^{\sigma_{1} \sigma_{2}}\left(k_{1}, k_{2}\right)\right. \\
& +G_{0}\left(k_{1}\right)_{n} G_{0}\left(k_{2}\right)_{n} T_{n n}^{\sigma_{1} \sigma_{2}}\left(k_{1}, k_{2}\right) \\
& \left.+2 G_{0}\left(k_{1}\right)_{p} G_{0}\left(k_{2}\right)_{n} T_{p n}^{\sigma_{1} \sigma_{2}}\left(k_{1}, k_{2}\right)\right],
\end{aligned}
$$

where exchange symmetry was employed for the last line.

Let us now consider the sum of diagrams c) and the isovector part of d) of fig.3. These diagrams consist of the pion-nucleon scattering in a two-nucleon reducible loop which is corrected by initial and final state interactions. The iterations are indicated by the ellipsis on both sides of the diagrams. In order to see that these diagrams cancel with eq.(5.4) let us take the diagram on the left hand side 
of fig. 4 with a twice iterated wiggly line vertex. It is given by [1]

$$
\begin{aligned}
\left(\Sigma_{c}+\sum_{d}^{i v}\right)^{L} & =i \varepsilon_{i j 3} \frac{q^{0}}{2 f^{2}}\left(1-g_{A}^{2} \frac{\mathbf{q}^{2}}{q_{0}^{2}}\right) \sum_{\alpha, \beta} \sum_{\sigma_{1}, \sigma_{2}} \int \frac{d^{4} k_{1}}{(2 \pi)^{4}} \frac{d^{4} k_{2}}{(2 \pi)^{4}} \\
& \times e^{i k_{1}^{0} \eta} e^{i k_{2}^{0} \eta} G_{0}\left(k_{1}\right)_{\alpha} G_{0}\left(k_{2}\right)_{\beta}\left[\frac{-i}{2} \int \frac{d^{4} q}{(2 \pi)^{4}}\right. \\
& \times \sum_{\alpha^{\prime}, \beta^{\prime}} \sum_{\sigma_{1}^{\prime}, \sigma_{2}^{\prime}} V_{\alpha \beta ; \alpha^{\prime} \beta^{\prime}}(q) \frac{\partial G_{0}\left(k_{1}-q\right)_{\alpha^{\prime}}}{\partial k_{1}^{0}} \tau_{\alpha^{\prime} \alpha^{\prime}}^{3} \\
& \left.\times V_{\alpha^{\prime} \beta^{\prime} ; \alpha \beta}(-q) G_{0}\left(k_{2}+q\right)_{\beta^{\prime}}\right]
\end{aligned}
$$

where the superscript $L$ indicates that the corresponding amplitude is calculated at the one-loop level. $V_{\alpha \beta ; \gamma \delta}$ corresponds to the wiggly line with the indices $\alpha$ and $\gamma$ belong to the out-/in-going first particle, in that order, and similarly $\beta$ and $\gamma$ for the second one. To shorten the notation, we have only indicated the isospin indices in $V$ in the previous equation, although $V$ depends also on spin. The derivative with respect to $k_{1}^{0}$ arises, because the nucleon propagator to which the two pions are attached appears squared [1]. This is so because for the $\pi^{ \pm}, i$ and $j$ can be either 1 or 2 , so that the only surviving contribution is $k=3$. For the $\pi^{0}, i=j=3$ and then there is no contribution. Thus, because one has either 0 or $\tau^{3}$, which is a diagonal matrix, the nucleon propagator before and after the two-pion vertex is the same. The $q$-loop integral in eq.(5.6) is typically divergent. Nevertheless, the parametric derivative with respect to $k_{1}^{0}$ can be extracted out of the integral as soon as it is regularized. Once this is done, the quantity between the squared brackets in eq.(5.6) corresponds to the twice iterated wiggly line contribution to $T_{N N}$. Of course, the same regularization method as that used to calculate $T_{N N}$ should be employed. In addition, the isovector nature of the modified WT vertex of eq.(5.1) implies that only the difference between the proton-proton and neutron-neutron contributions arises. This can be worked out straightforwardly from the isospin structure of the local four-nucleon vertex and that of the one-pion exchange [2]. As a result we can write

$$
\begin{aligned}
\left(\Sigma_{c}+\Sigma_{d}^{i v}\right)^{L} & =i \varepsilon_{i j 3} \frac{q^{0}}{2 f^{2}}\left(1-g_{A}^{2} \frac{\mathbf{q}^{2}}{q_{0}^{2}}\right) \sum_{\sigma_{1}, \sigma_{2}} \int \frac{d^{4} k_{1}}{(2 \pi)^{4}} \frac{d^{4} k_{2}}{(2 \pi)^{4}} \\
& \times e^{i k_{1}^{0} \eta} e^{i k_{2}^{0} \eta}\left[G_{0}\left(k_{1}\right)_{p} G_{0}\left(k_{2}\right)_{p} \frac{\partial}{\partial k_{1}^{0}} T_{p p}^{\sigma_{1} \sigma_{2}}\left(k_{1}, k_{2}\right)^{L}\right. \\
& \left.-G_{0}\left(k_{1}\right)_{n} G_{0}\left(k_{2}\right)_{n} \frac{\partial}{\partial k_{1}^{0}} T_{n n}^{\sigma_{1} \sigma_{2}}\left(k_{1}, k_{2}\right)^{L}\right]
\end{aligned}
$$

This result cancels exactly with that on the right hand side of fig.4, corresponding to the twice iterated wiggly line exchange contribution to $T_{N N}$ in eq.(5.4). This cancellation is explicit by reducing eq.(5.4) to the one-loop case for calculating $T_{N N}$. Notice as well that the contribution to $T_{N N}$ given by the exchange of only one wiggly line vanishes when inserted in eq.(5.4) because it is independent of $k_{1}^{0}$.

This process of mutual cancellation can easily be generalized to any number of two-nucleon reducible loops in figs.3a), b), c) and d). An $n+1$ iterated wiggly line exchange in these figures implies $n$ two-nucleon reducible
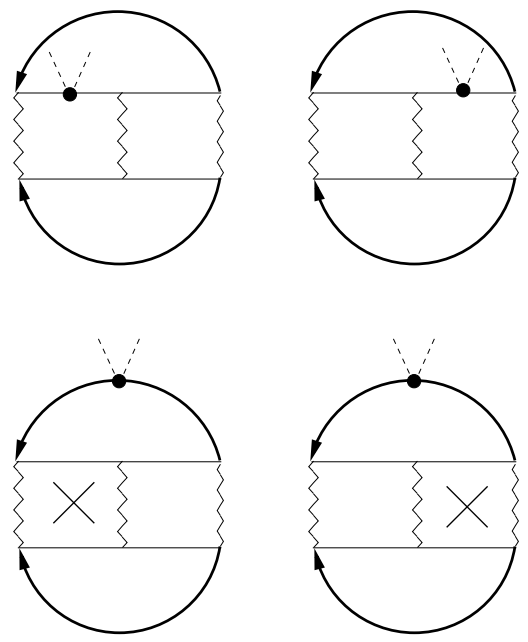

Fig. 5. In this figure the cross indicates the action of the derivative with respect to $k_{1}^{0}$ in eq.(5.7). When the derivative is performed over a baryon propagator the latter becomes squared. In this way, the first diagram on the second row of the figure is the same as the one to the left of the first row but with opposite sign and they cancel each other. The same applies to the second diagrams on the first and second rows.

loops. The two pions can be attached for $\Sigma_{c}+\Sigma_{d}^{i v}$ to any of them, while for $\Sigma_{a}+\Sigma_{b}^{i v}$ the derivative with respect to $k_{1}^{0}$ can also act on any of the loops (see fig. 5 for a thrice iterated wiggly line). The iterative loops are the same for both cases but a relative minus sign results from the loop on which the two pions are attached with respect to the one on which the derivative is acting, as just discussed. Hence,

$$
\Sigma_{a}+\Sigma_{c}=0, \quad \text { and } \quad \Sigma_{b}^{i v}+\Sigma_{d}^{i v}=0
$$

The basic simple reason for such cancellation is that while for $\Sigma_{a}+\Sigma_{c}^{i v}$ the presence of a nucleon propagator squared gives rise to $(-1)^{2} \partial / \partial k_{1}^{0}$, for $\Sigma_{b}+\Sigma_{d}^{i v}$ it yields $-\partial / \partial k_{1}^{0}$. The extra $(-1)$ for $\Sigma_{a}+\sum_{c}^{i v}$ results because it involves an integration by parts.

The same cancelation does not happen for the isoscalar contributions: for those diagrams, the derivative has vanished because the NLO term of the expansion of the poleterm propagator cancels the square of the propagator which closes the diagram. In diagram b) the "elementary" $\mathrm{NN}$ interaction $N_{J I}$ is dressed by an iterative process, whereas for diagram d) the NN amplitude is given by the pion scattering term corrected by initial and final state interactions.

\subsection{Unitary CHPT for in-medium nucleon-nucleon scattering [2]}

The evaluation of the NN scattering amplitudes in the nuclear medium can be obtained from results in the vacuum, since the only modification without increasing the chiral order corresponds to use the full in-medium nucleon propagators (4.1). This is directly accomplished by replacing 


\section{$19^{\text {th }}$ International IUPAP Conference on Few-Body Problems in Physics}

$$
\begin{aligned}
g \equiv L_{10, f} \longrightarrow L_{10} & =L_{10, f}+2 L_{10, m}+L_{10, d} \\
& =L_{10, p p}+L_{10, h h}
\end{aligned}
$$

in eq.(3.1), for any order of the NN scattering in the nuclear medium. We use the notation $L_{i j}$, where $i$ indicates the number of two-nucleon states and $j$ the number of pion exchanges in the loop. Also we use the subscripts $f, m$ and $d$ indicating zero, one or two Fermi sea insertions from the nucleon propagators in the medium. Using the particlehole representation of eq.(4.1) the loop integral (5.9) can also be written in terms of a particle-particle $\left(L_{10, p p}\right)$ and a hole-hole $\left(L_{10, h h}\right)$ part. The same process as discussed in subsec.3.1 is followed to fix $N_{J I}$, only that in all loop integrals the nucleon propagators have to be exchanged by their full in-medium equivalents.

The calculation of the diagrams c) and d) of fig. 3 in UCHPT requires some additional considerations. For those diagrams a pion scatters inside a two-nucleon reducible loop, where this loop has to be corrected by initial and final state interactions as denoted in the figure by the ellipsis. Then, instead of eq.(3.1) we have [2]

$H_{J I}(\ell, \bar{\ell}, S)=\sum_{\ell^{\prime}, \ell^{\prime \prime}} D_{J I}^{-1}\left(\ell, \ell^{\prime}, S\right) \cdot \xi_{J I}\left(\ell^{\prime}, \ell^{\prime \prime}, S\right) \cdot D_{J I}^{-1}\left(\ell^{\prime \prime}, \bar{\ell}, S\right)$,

where $D_{J I}=I+N_{J I} L_{10}$. The LO result requires to employ $D_{J I}^{(0)}$ and to calculate the two-nucleon reducible loop to which the two pions are attached by factorizing on-shell the NN scattering amplitudes. We use the notation $D_{J I}^{(n) ; i_{3}}=$ $I+N_{J I}^{(n) ; i_{3}} L_{10}^{i_{3}}$ with $n$ the chiral order,

$$
\begin{aligned}
\left.H_{J I}\right|_{L O} & =D_{J I}^{(0)-1} \cdot \xi_{J I}^{(0)} \cdot D_{J I}^{(0)^{-1}}, \\
\xi_{J I}^{(0)} & =-\left(N_{J I}^{(0)}\right)^{2} \cdot D L_{10},
\end{aligned}
$$

where $D L_{10}$ represents the term of the pion scattering inside a two-nucleon reducible loop, which is

$$
D L_{10 ; i v}=-i \varepsilon_{i j 3} \frac{m q^{0}}{f^{2}}\left(1-g_{A}^{2} \frac{\mathbf{q}^{2}}{q_{0}^{2}}\right) i_{3} \frac{\partial L_{10}^{i_{3}}}{\partial A},
$$

for the isovector part. UCHPT reproduces the cancelation of the isovector parts, as generally shown in the last subsection. For explicit results and further discussion we just refer the interested reader to ref.[2].

\section{Conclusions and outlook}

We have reviewed the development in refs. $[1,2]$ of a power counting in the nuclear medium that combines both shortrange and pion-mediated inter-nucleon interactions. The power counting requires typically the resummation of infinite strings of two-nucleon reducible diagrams with the leading $O\left(p^{0}\right)$ two-nucleon CHPT amplitudes. One possible explicit method for performing those resummations, called UCHPT, has been presented. The pion self-energy in asymmetric nuclear matter has been calculated up-toand-including $O\left(p^{5}\right)$. It is shown on general grounds that the leading corrections to the linear density approximation vanish. In particular, it is derived that the leading corrections from NN scattering mutually cancel. More calculations for other physical processes and higher orders are clearly needed to assess the realm of applicability of the present approach. As a most recent development in ref.[2] the main trends for the energy density of symmetric nuclear and neutron matter has already been reproduced at next-to-leading order.

\section{References}

1. J. A. Oller, A. Lacour and U.-G. Meißner, J. Phys. G: Nucl. Part. Phys. 37 (2010) 015106 [arXiv:nuclth/0902.1986].

2. A. Lacour, J. A. Oller and U.-G. Meißner, arXiv:0906.2349 [nucl-th].

3. S. Weinberg, Physica A 96 (1979) 327.

4. S. Weinberg, Phys. Lett. B 251 (1990) 288.

5. S. Weinberg, Nucl. Phys. B 363 (1991) 3.

6. E. Epelbaum, H. W. Hammer and U.-G. Meißner, Rev. Mod. Phys., in print, arXiv:0811.1338 [nucl-th].

7. R. J. Furnstahl, G. Rupak and T. Schafer, Ann. Rev. Nucl. Part. Sci. 58 (2008) 1 [arXiv:nuclth/0801.0729], and references therein.

8. J. A. Oller and E. Oset, Phys. Rev. D 60 (1999) 074023 [arXiv:hep-ph/9809337].

9. J. A. Oller and U.-G. Meißner, Phys. Lett. B $\mathbf{5 0 0}$ (2001) 263 [arXiv:hep-ph/0011146].

10. M. Ericson and T. E. O. Ericson, Annals Phys. 36 (1966) 323.

11. E. Friedman and A. Gal, Phys. Rept. 452 (2007) 89, and references therein.

12. U.-G. Meißner, J. A. Oller and A. Wirzba, Annals Phys. 297 (2002) 27.

13. G. Chanfray, M. Ericson and M. Oertel, Phys. Lett. B 563 (2003) 61.

14. M. Döring and E. Oset, Phys. Rev. C 77 (2008) 024602.

15. N. Kaiser and W. Weise, Phys. Lett. B 512 (2001) 283.

16. T. S. Park, H. Jung and D. P. Min, J. Korean Phys. Soc. 41 (2002) 195.

17. L. Girlanda, A. Rusetsky and W. Weise, Nucl. Phys. A 755 (2005) 653.

18. J. A. Oller, Phys. Rev. C 65 (2002) 025204.

19. V. Bernard, N. Kaiser and U.-G. Meißner, Int. J. Mod. Phys. E 4 (1995) 193.

20. A. L. Fetter and J. D. Walecka, "Quantum Theory of Many-Particle Systems". Dover Publications, Inc., Mineola, New York. 1971 edition. 